\title{
Linear and Circular Polarization Properties of Jets *
}

\author{
Mateusz Ruszkowski \\ JILA, University of Colorado at Boulder, CO 80309, USA
}

September 30, 2002

\begin{abstract}
I discuss the transfer of polarized synchrotron radiation in relativistic jets. I argue that the main mechanism responsible for the circular polarization properties of compact synchrotron sources is likely to be Faraday conversion and that, contrary to common expectation, a significant rate of Faraday rotation does not necessarily imply strong depolarization. The long-term persistence of the sign of circular polarization, observed in some sources, is most likely due to a small net magnetic flux generated in the central engine, carried along the jet axis and superimposed on a highly turbulent magnetic field. I show that the mean levels of circular and linear polarizations depend on the number of field reversals along the line of sight and that the gradient in Faraday rotation across turbulent regions can lead to "correlation depolarization". The model is potentially applicable to a wide range of synchrotron sources. In particular, I demonstrate how the model can naturally explain the excess of circular over linear polarization in the Galactic Center $\left(\operatorname{Sgr} \mathrm{A}^{*}\right)$ and the low-luminosity AGN M81*.
\end{abstract}

Keywords: polarization: circular, linear - Galaxy: center - galaxies: individual (M81) - quasars: individual (3C279)

\section{Introduction}

Polarization has proven to be an important tool in AGN research. In principle, linear and particularly circular polarization observations of synchrotron radiation may permit measurements of various properties of relativistic jets such as: magnetic field strength and topology, the net magnetic flux carried by jets (and hence generated in the central engine), the energy spectrum of radiating particles, and the jet composition (i.e., whether jets are mainly composed of $e^{+}-e^{-}$ pairs or electron-proton plasma). The renewed interest in polarization of compact radio sources stems from two recent developments. First, Bower et al. (1999) detected circular polarization using the Very Large Array (VLA) in the best supermassive black hole candidate, the Galactic Center (Sgr A*). This discovery was quickly confirmed by Sault and Macquart (1999) using the Australia Telescope Compact Array (ATCA). Circular polarization was also detected in the celebrated X-ray binary system SS 433 (Fender et al., 2000) and the microquasar GRS $1915+105$ (Fender et al., 2002). Moreover, the Very

\footnotetext{
* Invited talk
}

(c) 2018 Kluwer Academic Publishers. Printed in the Netherlands. 
Long Baseline Array (VLBA) has now detected circular polarization in as many as 20 AGN (Wardle et al., 1998; Homan and Wardle, 1999). Second, it is now possible to measure circular polarization with an unprecedented accuracy of $0.01 \%$ using the ATCA (Rayner et al., 2000). This dramatic improvement in the observational status of polarization measurements has also brought new questions. For example, there is observational evidence that the sign of circular polarization is persistent over decades in some sources (Komesaroff et al., 1984; Homan and Wardle, 1999; Hughes, 2002), which indicates that it is a fundamental property of jets and strongly suggests that a small magnetic flux is

frozen into jets. Another problem, is how to reconcile the high level of circular polarization with the lower value of linear polarization in Sgr A* (Bower et al., 1999) and M81* (Brunthaler et al., 2001; Bower, Falcke and Mellon, 2002). Moreover, there is not even a general consensus on the mechanism responsible for the circular polarization properties of jets (Wardle et al., 1998).

\section{Observational trends}

Compact radio sources typically show a linear polarization (LP) of a few percent of the total intensity (Jones et al., 1985). This is much less than the theoretical maximum for synchrotron sources, which can approach $70 \%$ in homogeneous sources with unidirectional magnetic field. Therefore, magnetic fields in radio sources are believed to be highly inhomogeneous, although the nonvanishing linear polarization is in itself an indirect indication of a certain degree of ordering of the field. From the theoretical point of view, ordered jet magnetic field is expected when shocks compress an initially random field (Laing, 1980; Laing, 1981; Hughes et al., 1989) or when such initial fields are sheared along the jet (Laing, 1980; Laing, 1981; Begelman, Blandford and Rees, 1984).

Circular polarization $(\mathrm{CP})$ is a common feature of quasars and blazars (Rayner et al., 2000; Homan et al., 2001). It is usually characterized by an approximately flat spectrum, and is generated near synchrotron selfabsorbed jet cores (Homan and Wardle, 1999). CP is detected in about $30 \%-50 \%$ of these objects. Measured degrees of CP are generally lower than the levels of LP and usually range between $0.1 \%$ and $0.5 \%$ (Homan and Wardle, 1999; Homan et al., 2001). Observations of proper motion of CP-producing regions in the quasar 3C 273 (Homan and Wardle, 1999) suggest that circular polarization is intrinsic to the source, as opposed to being due to foreground effects. 


\section{Mechanisms for producing circular polarization}

The most obvious candidate for generating circular polarization in compact radio sources is intrinsic emission (Legg and Westfold, 1968). However, intrinsic CP will be strongly suppressed by the tangled magnetic field and possibly by $e^{+}-e^{-}$pairs, which do not contribute CP. Other mechanisms have also been proposed, among which the most popular ones are scintillation (Macquart and Melrose, 2000), general relativistic effects in dispersive plasma (Broderick and Blandford, 2002) and Faraday conversion (Jones and O'Dell, 1977a; Jones, 1988; Wardle et al., 1998; Ruszkowski and Begelman, 2002; Beckert and Falcke, 2002). The scintillation mechanism, in which circular polarization is stochastically produced by a birefringent screen located between the jet and the observer, fails to explain the persistent sign of circular polarization (if required by observations in a given source) as the time-averaged CP signal is predicted to vanish. The second mechanism can be important close to the central black hole. The last mechanism - Faraday conversion - is a very promising one and in the next subsection I discuss it in more detail.

\subsection{FARADAY ROTATION AND CONVERSION}

The polarization of radiation changes as it propagates through any medium in which modes are characterized by different plasma speeds. In the case of plasma dominated by cold electrons the modes are nearly circularly polarized. The left and right circular modes have different phase velocities and therefore the linear polarization vector of the propagating radiation rotates. This effect - Faraday rotation - is a specific example of a more general phenomenon called birefringence. In a medium whose natural modes are linearly or elliptically polarized, such as a plasma of relativistic particles, birefringence leads to the partial cyclic conversion between linearly and circularly polarized radiation as the phase relationships between the modes along the ray change with position.

\subsubsection{Strong rotativity}

Strong departures from mode circularity occur only when radiation propagates within a small angle $\sim \nu_{L} / \nu$ of the direction perpendicular to the magnetic field, where $\nu_{L}=e B / 2 \pi m_{e} c$ (quasi-transverse limit, QT). Therefore radiative transfer is often performed in the quasilongitudinal (QL) approximation. In a typical observational situation it is usually assumed that Faraday rotation within the source cannot be too large, as this will lead to the suppression of linear polarization. 


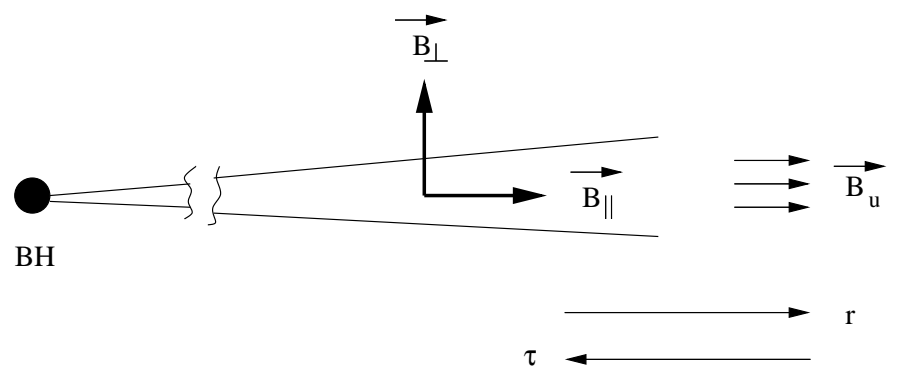

Figure 1. Jet and its magnetic field (left panel) and geometry of the assumed magnetic field assumed in the calculations (right panel).

However, this constraint does not prevent rotativity from achieving large values locally as long as the mean rotativity, i.e., averaged over all directions of magnetic field along the line of sight, is indeed relatively small. Such a situation may happen in a turbulent plasma. Technically, the strong rotativity regime $\left(\zeta_{v}^{* 2} \gg \zeta_{q}^{* 2}\right.$, see below) is equivalent to the QL limit (Jones and O'Dell, 1977b) and in this proceedings I mostly focus on this approximation.

\section{Model for polarization}

I consider a highly tangled magnetic field with a very small mean component which is required to determine the sign of circular polarization. From a theoretical view-point, we would expect some net poloidal magnetic field, either originating from the central black hole or from the accretion disk, to be aligned preferentially along the jet axis. Specifically, from equipartition and flux freezing arguments applied to a conical jet (Blandford and Königl, 1979) we get $\left\langle B_{\|}^{2}\right\rangle^{1 / 2} \sim\left\langle B_{\perp}^{2}\right\rangle^{1 / 2} \sim$ $B_{\text {rms }} \propto r^{-1}$ where $r$ is the distance along the synchrotron emitting source and the symbols $\|$ and $\perp$ refer to magnetic fields parallel and perpendicular to the jet axis, respectively. From the flux-freezing argument applied to the small parallel bias in the magnetic field we obtain $\left\langle B_{\perp}\right\rangle \sim 0$ and $\left\langle B_{\|}\right\rangle \propto r^{-2} \propto \delta B_{\mathrm{rms}}$, where $\delta \equiv B_{u} / B_{\mathrm{rms}} \ll 1$ is the ratio of the uniform and fluctuating components of the magnetic field (see Figure 1).

\subsection{Mean Stokes Parameters in the PRESEnCE of Field REVERSALS}

I solve the radiative transfer of polarized radiation in a turbulent plasma by adopting transfer equations for a piecewise homogeneous medium 


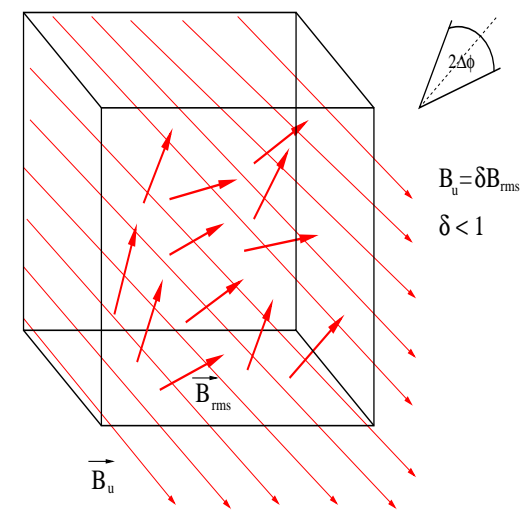

Figure 2. Jet and its magnetic field (left panel) and geometry of the assumed magnetic field assumed in the calculations (right panel).

with a weakly anisotropic dielectric tensor (Sazonov, 1969; Jones and O'Dell, 1977a). I assume that the mean rotation per unit synchrotron optical depth $\left\langle\zeta_{v}^{*}\right\rangle \equiv \delta \zeta$ and that azimuthal angle $\phi \in[-\Delta \phi, \Delta \phi]$, polar angle $\theta \in[0, \pi],\langle\sin 2 \phi\rangle=0$, and $\langle\cos 2 \phi\rangle=2 p-1$, where $0 \leq p \leq 1$ is a parameter describing the polarization direction and degree of order in the field (see Figure 2). In the case when synchrotron depth is large and the magnetic field unidirectional and appropriately rotated, the transfer equation has a particularly simple form:

$$
\left(\begin{array}{cccc}
1 & \zeta_{q} & 0 & 0 \\
\zeta_{q} & 1 & \zeta_{v}^{*} & 0 \\
0 & -\zeta_{v}^{*} & 1 & \zeta_{q} \\
0 & 0 & -\zeta_{q}^{*} & 1
\end{array}\right)\left(\begin{array}{c}
I \\
Q \\
U \\
V
\end{array}\right)=\left(\begin{array}{c}
1 \\
\epsilon_{q} \\
0 \\
0
\end{array}\right) J
$$

where the orientation-dependent $J$ and $\zeta_{q}, \zeta_{q}^{*}, \zeta_{v}^{*}$ are the source function, Q-absorptivity, convertibility and rotativity, respectively. In a realistic situation, magnetic field will not be uniform. From the analytic view-point, polarization can be calculated by averaging equation of radiative transfer over many orientations of magnetic field. Then, the levels of polarization depend on the mean products of Stokes parameters and rotativity:

$$
\left\langle\zeta_{v}^{*} U\right\rangle=\delta \zeta_{v}^{*}\langle U\rangle+\left\langle\widetilde{\zeta}_{v}^{*} \widetilde{U}\right\rangle
$$

where the correlation term $\left\langle\widetilde{\zeta}_{v}^{*} \widetilde{U}\right\rangle \propto N^{-1}$, where $N$ is the number of field reversals along the line of sight. The correlation term leads to depolarization when $N$ becomes sufficently small. Heuristically, when $N$ is large then, as the radiation propagates through turbulent zones, the polarization vector fluctuates to a lesser degree then when $N$ is 
small. In the later case, projected orientation of the polarization is more chaotic and the source is depolarized.

\subsection{FidUCIAL MODEL}

I assume that the typical mean Lorentz factor of radiating electrons $\gamma \sim 10^{2}$ and that the electron energy distribution function has a powerlaw form $n \propto \gamma^{-(2 \alpha+1)}$, where $\alpha$ is the spectral index of optically thin synchrotron emission. I use $\alpha=0.5$ and assume that the electron distribution is cut-off below $\gamma_{i} \sim$ a few. For example, for the maximum brightness temperature $T_{b} \sim 10^{11} \mathrm{~K}$ (Readhead, 1994) we have $\gamma \sim$ $3 k T_{b} / m_{e} c^{2} \sim 50$, which corresponds to mean rotation and conversion per unit synchrotron optical depth of order $\sim \delta \zeta_{v}^{*} \sim 3 \times 10^{3} \delta \ln \gamma_{i} / \gamma_{i}^{3}$ and $\zeta_{q}^{*} \sim-\ln \left(\gamma / \gamma_{i}\right)$, respectively, for $\nu \sim \gamma^{2} e B / 2 \pi m_{e} c$. Results from the simulations are shown in Figure 4.

Note that, even though the rotation per unit depth is very large and the magnetic field points (almost) randomly away and towards the observer, the source is not totally depolarized. Note also that, circular polarization peaks around $\tau \sim 1$. CP may change sign if $\tau$ varies strongly from optically thin to optically thick regime. Linear polarization does tend to zero when the mean rotativity $\left(\propto \delta \propto B_{u}\right)$ is high. Thus CP, which is produced by conversion of LP, also decreases for high $\delta$. (see Figure 3 and its caption for additional explanation).

\subsection{Quasar 3C279}

Wardle et al. (1998) reported the discovery of circular and linear polarization in 3C 279 and attributed CP to internal Faraday conversion. Typical fractional linear and circular polarizations in 3C 279 are of order $\sim 10 \%$ and $>1 \%$, respectively. They concluded that if the jet is

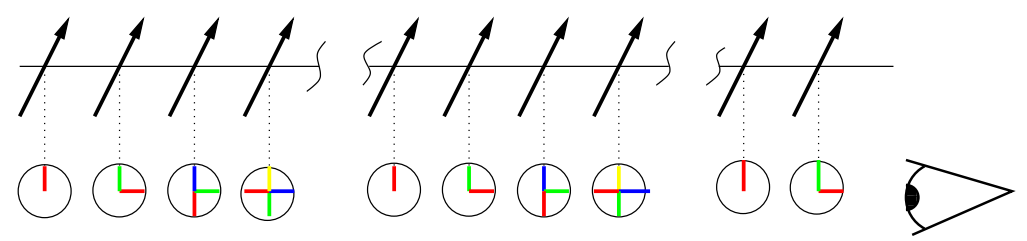

Figure 3. This plot demonstrates how a uniform magnetic field can depolarize most of the intrinsically polarized radiation when emission is present along the line of sight. Arrows indicate uniform magnetic field. Circular diagrams show the orientation of the polarization vector. Locally generated linear polarization is always denoted by a vertical line (i.e., 12 o'clock). Linearly polarized flux from the neighboring regions cancels out. The observed polarized radiation is generated in the regions closest to the observer. 

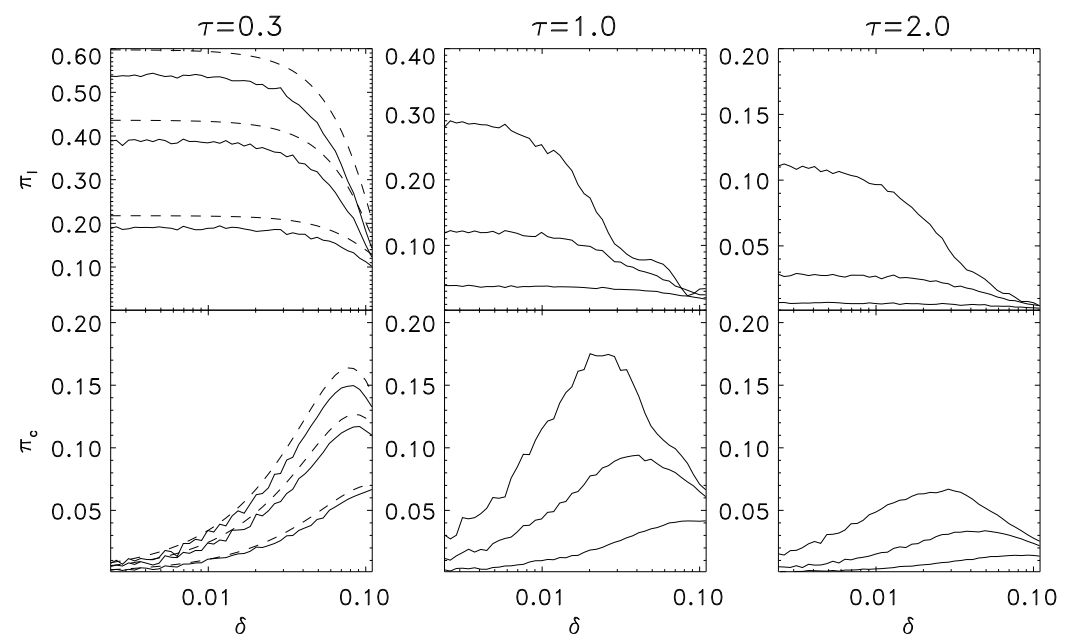

Figure 4. Linear and circular polarization as a function of the ratio of the uniform to fluctuating components of magnetic field $\delta=B_{u} / B_{r m s}$ for different synchrotron depths (upper labels). Curves within each panel correspond to different numbers of field reversals $N$ (for smaller $N$ polarization levels are lower).

composed of normal plasma, then the low-energy cut-off of the energy distribution of relativistic electrons must be as high as $\gamma_{i} \sim 100$ in order to avoid Faraday depolarization and overproduction of the jet kinetic power. They were unable to fit their polarization models to the observational data for $\gamma_{i}>20$ and thus claimed that the jet must be pair-dominated. However, the above observational constraints on $\mathrm{CP}$ and LP and the jet energetics can be satisfied for a variety of microscopic plasma parameters. This is due to the fact that different "microscopic" parameters, such as $\gamma_{i}$, the ratio of the cold to relativistic electron number densities, or the positron fraction, can lead to similar "macroscopic" parameters such as convertibility and rotativity. In order to illustrate this, I consider two radically different examples and show that both cases can lead to the same CP and LP.

\subsubsection{Electron-proton jet}

In this example, plasma is composed exclusively of a mixture of protons and electrons with both relativistic (r) and cold (c) populations being present. For a low-energy cut-off $\gamma_{i} \sim 30$ and an electron number density-weighted mean Lorentz factor $\gamma \sim 50$ and $\alpha=0.5$, we get $\left\langle\zeta_{v}^{*}\right\rangle \sim 80\left(n_{c} / n_{r}\right) \delta$ and $\left\langle\zeta_{q}^{*}\right\rangle \sim-0.5$. For the above choice of parameters, the main contribution to rotativity comes from cold electrons as long as $n_{c} / n_{r}>5 \times 10^{-3}$. The required levels of LP and CP can be obtained, for example, when $\tau=1, \alpha=0.5, N=15,2 \Delta \phi=35^{\circ}, \delta \sim 2.5 \times 10^{-1}$ 
and $\left(n_{c} / n_{r}\right)=7 \times 10^{-2}$. Bear in mind that the admixture of cold electrons does not have to be large to explain the data. Interestingly, a jet with such a plasma composition could carry roughly as small a kinetic power as the pure electron-positron jet with the same emissivity, since the ratio of kinetic powers of an $e-p$ jet to a pure relativistic $e^{+}-e^{-}$ jet is $\sim 18.4\left(\langle\gamma\rangle_{e^{+} e^{-}} / 50\right)^{-1}\left(\gamma_{i, e^{+} e^{-}} / \gamma_{i, p e}\right)$.

\subsubsection{Electron-positron jet}

The alternative possibility is that the jet is dominated by relativistic pair plasma. For example, for $\gamma_{i}=2$ and $\gamma=50$ we get $\left\langle\zeta_{q}^{*}\right\rangle \sim-3.1$ and $\left\langle\zeta_{v}^{*}\right\rangle \sim 1.4 \times 10^{2} \delta\left(n_{p} / n_{e-}\right)$. Agreement with the observed fractional LP and $\mathrm{CP}$ can be obtained for example when the jet is pair-dominated in the sense that $n_{e-} \gg n_{p}$, while being dominated dynamically by protons. I was able to obtain the required polarization levels in this case for $\tau=1, N=40,2 \Delta \phi=30^{\circ}, \delta \sim 0.3$ and $n_{p} / n_{e-}=0.1$. Recent theoretical work of (Sikora and Madejski, 2000) suggests that jets may be pair-dominated numberwise but still dynamically dominated by protons.

\subsection{Galactic Center - Sgr A*}

Recently (Bower et al., 1999) reported the detection of circular polarization from our Galactic Center $\left(\mathrm{Sgr} \mathrm{A}^{*}\right)$ with the VLA, which was confirmed by (Sault and Macquart, 1999) using ATCA. The typical level of $\mathrm{CP}$ in their observations was $\sim 0.3 \%$, greater than the level of linear polarization. This result may seem surprising in light of the strong limits on the ratio of $\mathrm{CP}$ to $\mathrm{LP}$ in AGN where $\mathrm{CP} / \mathrm{LP}$ is usually much less than unity. However, as explained above, an excess of CP over LP can be explained easily in the framework of our model. Archival VLA data indicate that the mean CP was stable over ten years (Bower, Falcke and Mellon, 2002). This is also not surprising as our model naturally predicts a persistent $\mathrm{CP}$ sign. The average CP spectrum was characterized by a flat to slightly positive spectral index $\left(\pi_{c} \propto \nu^{\beta}, \beta>0\right)$. This result can also be accounted for in our model. For example, in the framework of a self-absorbed, self-similar jet model (Blandford and Königl, 1979) but with a small bias $\delta$ we have $\left\langle\zeta_{v}^{*}\right\rangle \propto \delta \propto B \propto \nu \propto r^{-1}$ (see Figure 1). The minimum and maximum size of Sgr $\mathrm{A}^{*}$ constrain the brightness temperature to be $10^{10}<T_{b}<5 \times 10^{11} K$ (Melia and Falcke, 2001), which is within the range of typical AGN radio cores. Taking $T_{b} \sim 10^{11}$ as the representative rest frame value (Readhead, 1994), we get $\gamma \sim 50$. The required levels of CP and LP can be approximatelly obtained, for example, for $\tau=1, N=45, p=1, \alpha=0.5, \gamma_{i}=3, \gamma \sim 50$ and $\delta=0.35$ (see Figure 
5).

It has recently been suggested that observations of linear polarization can be used to constrain the accretion rate in Sgr $\mathrm{A}^{*}$ and other low-luminosity AGN (Agol, 2000; Quataert and Gruzinov, 2000). These authors base their argument on the assumption that the Faraday rotation measure has to be sufficiently small in order not to suppress strong linear polarization at higher frequencies (Aitken et al., 2000; Bower et al., 2002). This assumption places limits on density and magnetic field strength and leads to very low accretion rates $\sim 10^{-8}$ to $10^{-9} M_{\odot} \mathrm{yr}^{-1}$. High values of linear polarization at higher frequencies may originate closer to the central black hole, where the bulk of the LP emitting material may no longer be in the form of a self-absorbed jet but rather in the form of an accretion disk. As noted by Agol (2000) and Quataert and Gruzinov (2000), the above accretion rate is inconsistent with an advection-dominated model for Sgr $\mathrm{A}^{*}$, which assumes that the accretion rate is of order the canonical Bondi rate $\sim 10^{-4}$ to $10^{-5} M_{\odot} \mathrm{yr}^{-1}$. However, strong rotation measure does not in principle limit densities and magnetic fields provided that the field has a small magnetic flux associated with it, which is required to define the sign of circular polarization (note that the rotation angle is then reduced $\delta^{-1}$ times).

\subsection{Low-Luminosity AGN - M81*}

Circular polarization was also detected in the compact radio jet of the nearby spiral galaxy M81 (Brunthaler et al., 2001). Their estimated values of $\mathrm{CP}$ were $0.27 \pm 0.06 \pm 0.07 \%$ at $4.8 \mathrm{GHz}$ and $0.54 \pm 0.06 \pm 0.07 \%$ at $8.4 \mathrm{GHz}$, where errors are separated into statistical and systematic terms (see also Bower et al. 2002 for more results on LLAGN). This suggests that the $\mathrm{CP}$ spectrum is flat or possibly inverted. They also detected no linear polarization at a level of $0.1 \%$, indicating that the source has a high circular-to-linear polarization ratio. The spectral index indicates that this source is synchrotron self-absorbed and we can apply the same approach as for Sgr A*.

\section{Conclusions}

I have considered the transfer of polarized synchrotron radiation in jets and have argued that Faraday conversion is the primary mechanism responsible for the circular polarization properties of compact radio sources. The modelis potentially applicable to a wide range of sources. A crucial ingredient of the model is a small bias in the highly turbulent magnetic field which accounts for the persistence of the sign of circular 


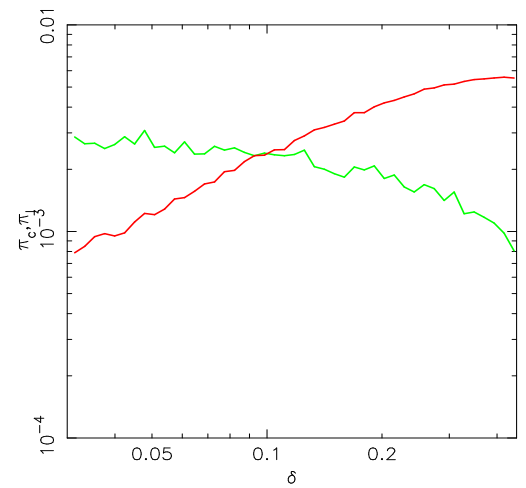

Figure 5. Linear (decreasing curve) and circular polarization as a function of $\delta$ for Sgr A* (intrinsic CP included)

polarization. This bias is a direct evidence for the net magnetic flux carried by magnetically accelerated jets (Blandford and Payne, 1982; Li et al., 1992).

Extremely large Faraday rotation per unit synchrotron absorption depth, does not necessarily lead to depolarization provided that the mean rate of Faraday rotation across the source is relatively small, or in other words, that the turbulent magnetic field is characterized by a small magnetic flux. Indeed, a large Faraday rotativity is required in order to explain the high ratio of circular to linear polarization observed in some sources (Galactic Center, M81*). Constraints on jet composition or accretion rate, based on the requirement that the source does not become Faraday depolarized, may be circumvented under these conditions.

Gradients in Faraday rotation across turbulent cells can lead to correlations between rotativity and Stokes $Q$ and $U$ parameters, which can result in "correlation depolarization". Variations in the mean parameters are unlikely to change the helicity of circular polarization unless a source undergoes a sharp transition from very low to very high synchrotron depth.

\section{Acknowledgements}

I thank Mitch Begelman for collaboration on this project. I also thank Don Backer, Roger Blandford, Geoff Bower, Avery Broderick, Rob Fender, Dan Homan, Marek Sikora and John Wardle for insightful discussions. I am also grateful to the organizers for organizing a very 
interesting conference. This work was supported in part by NSF grant AST-9876887.

\section{References}

Agol, E. 2000, ApJ, 538, L121

Aitken, D.K., Greaves, J., Chrysostomou, A., Jennes, T., Holland, W., Hough, J.H., Pierce-Price, D, and Richer, J. 2000, ApJ, 534, L173

Beckert, T., and Falcke, H. 2002, A\&A, 388, 1106

Begelman, M.C., Blandford R.D., and Rees, M.J. 1984, Rev. Mod. Phys., 56, 255

Blandford, R.D., and Königl, A. 1979, ApJ, 232, 34

Blandford, R.D., and Payne, D.G. 1982, MNRAS, 199, 883

Bower, G.C., Falcke, H., and Backer, D.C. 1999, ApJ, 523, L29

Bower, G.C., Falcke, H., Sault, H., and Backer, D.C. 2002, ApJ, 571, 843

Bower, G.C., Falcke, H., and Mellon, R.R. 2002, ApJL, in press

Bower, G.C., Wright M.C.H., Falcke, H., and Backer, D.C. 2002, ApJ, submitted Broderick, A., and Blandford, R., these proceedings

Brunthaler, A., Bower, G.C., Falcke, H., and Mellon, R.R. 2001, ApJL, 560, 123

Fender, R., Rayner D., Norris R., Sault R.J., and Pooley, G. 2000, ApJ, 530, L29

Fender, R., et al. 2002, MNRAS, 336, 39

Homan, D.C., and Wardle, J.F.C. 1999, AJ, 118, 1942

Homan, D.C., Attridge, J.M., and Wardle J.F.C. 2001, ApJ, 556, 113

Hughes, P.A., Aller H.D., and Aller M.F. 1989, ApJ, 341, 54

Hughes, P.A. 2002, these proceedidings

Jones, T.W. 1988, ApJ, 332, 678

Jones, T.W., and O'Dell S.L. 1977, ApJ, 214, 522

Jones, T.W., and O'Dell S.L. 1977, ApJ, 215, 236

Jones, T.W., Rudnick, L., Aller, H.D., Aller, M.F., Hodge, P.E., and Fiedler, R.L. 1985, ApJ, 290, 627

Komesaroff, M.M., Roberts, J.A., Milne, D.K., Rayner, P.T., and Cooke, D.J. 1984, MNRAS, 208, 409

Laing, R.A. 1980, MNRAS, 193, 493

Laing, R.A. 1981, ApJ, 248, 87

Legg, M.P.C., and Westfold, K.C. 1968, ApJ, 154, 499

Li Z-Y., Chiueh, T., and Begelman M.C. 1992, ApJ, 394, 459

Macquart, J.-P., and Melrose, D.B. 2000, ApJ, 545, 798

Melia, F., and Falcke, H. 2001, ARA\&A, 39, 309

Quataert, E., and Gruzinov, A. 2000, ApJ, 545, 842

Rayner, D.P., Norris, R.P., and Sault, R.J. 2000, MNRAS, 319, 484

Readhead, A.C.S. 1994, ApJ, 426, 51

Ruszkowski, M., and Begelman M.C. 2002, ApJ, 573, 485

Sault, R.J., and Macquart, J.-P. 1999, ApJ, 526, L85

Sazonov, V.N. 1969, Sov. Phys. JETP, 29, 578

Sikora, M., and Madejski, G. 2000, ApJ, 534, 109

Wardle, J.F.C., Homan, D.C., Ojha, R., and Roberts, D.H. 1998, Nature, 395, 457 
cpmr.tex; 24/12/2018; 23:27; p.12 